\title{
Evolution der Krankenrolle - eine These
}

Thomas Knecht

Korrespondenz:

Dr. med. Thomas Knecht

Leitender Arzt

Psychiatrische Klinik

Postfach 154

CH-8596 Münsterlingen

Tel. 0716864141

Fax 0716864035

thomas.knecht@stgag.ch
Nicht erst seit dem Darwin-Jahr 2009, das heisst 150 Jahre nach dem Erscheinen von «Entstehung der Arten», wird man sich inne, dass evolutionäre Mechanismen nicht nur in Fauna und Flora, sondern im gesamten bekannten Kosmos am Werk sind [1]. Das Prinzip von Variation und Selektion der überlebenstüchtigsten, d.h. der bestangepassten Variante lässt sich offenbar bis weit in den Mikro- und Makrokosmos hinein ausdehnen, scheint geradezu ubiquitär zu sein. Forscher wie Wojciech H. Zurek [2] machen geltend, dass bereits auf der Ebene der scheinbar anarchischen quantenmechanischen Zustände eine grosse Variationsbreite besteht, wobei unterschiedlich «fitte» Varianten unterschiedliche Chancen haben, zu einem stabilen Zustand (sog. pointer states) zu finden, sich so in der Zeit fortzupflanzen und damit Teil der klassischen Welt zu werden. Mittlerweile haben physikalische Experimente diesen «Quantendarwinismus» bestätigt.

Am anderen Ende der Grössenordnungen ist es der theoretische Physiker Lee Smolin [3], der ein Konzept der «kosmologischen Vererbung» entworfen hat. Seine Theorie ist mangels empirischer Überprüfbarkeit noch nicht allgemein etabliert, steht aber in guter Übereinstimmung mit der Stringtheorie (die ihrerseits noch unbelegt ist), weshalb sie in Fachkreisen durchaus ernsthaft diskutiert wird. Smolin führt die Vorstellung ein, dass unser Universum nur eines von verschiedenen Paralleluniversen ist («Multiversum»), allerdings dasjenige, das aufgrund optimaler Gewichtung seiner Naturkonstanten (Teilchenmassen, Gravitationsstärke usw.) seine Überlebensfähigkeit schlüssig unter Beweis gestellt hat.

Nun scheint es auch in unseren mesokosmischen Verhältnissen möglich, die fundamentalen Prinzipien der Evolution breiter anzuwenden, nämlich auch auf den Bereich der soziokulturellen Entwicklungen und Errungenschaften. Dieser Vorschlag wurde von Richard Dawkins [4] bereits Mitte der 70er Jahre gemacht. Ganz in Analogie zur Genetik schlug Dawkins vor, die reproduktionsfähige Informationseinheit, gewissermassen das «Kulturgen», als «Mem» zu bezeichnen. Die Lehre von Inhalt und Verbreitung der Meme ist demnach die Memetik, die von Susan Blackmore in ihrem Schlüsselwerk aus dem Jahr 2000 [5] leicht verständlich dargestellt wird.

Einen Diskurs über soziokulturelle Evolution zu führen, ist indessen ein heikles Unterfangen, zumal evolutionäre Ansätze, wie sie im 19. Jahrhundert gepflegt wurden, aufgrund ihrer wertenden Unterscheidung zwischen «primitiven» und «zivilisierten» Kulturen von den Sozialwissenschaftern des 20. Jahrhunderts der Unwissenschaftlichkeit bezichtigt wurden.

\section{Zusammenfassung}

In diesem Beitrag geht der Autor der Frage nach, inwieweit Patientenverhalten einen Anpassungsprozess im Wandel der Zeit durchläuft. Dabei zeigt sich, dass sowohl die Krankenrolle als auch die Krankenfürsorge Produkte einer kulturellen Co-Evolution sind, die sich gegenseitig stark beeinflussen. Die Stufen dieser durchaus gerichteten Entwicklung widerspiegeln die bekannten Evolutionsstufen des Altruismus: Nepotismus, Parochialismus, reziproker Altruismus. Die kulturelle Evolution ist dabei allerdings nicht stehengeblieben: Das Solidaritätsprinzip, das durch die Schaffung der Sozialwerke in die Welt trat, entlastet den Einzelnen massiv gegenüber der Gemeinschaft. Aber auch damit ist offenbar noch kein Endpunkt erreicht: Ein «Hypersolidarismus», der die Einzelinteressen noch höher gewichtet und dem Individuum zunehmend jede reziproke Verpflichtung gegenüber dem Kollektiv erspart, könnte sich als kulturrevolutionärer Auswuchs erweisen und damit längerfristig das Bestehen der Sozialwerke gefährden.

Dies nicht zuletzt aus dem Grund, dass solche Betrachtungsweisen auch zur Rechtfertigung von Sklaverei, Kolonialismus und Eugenik herangezogen wurden (vgl. Sozialdarwinismus, Rassenhygiene). So galt es fortan als illegitim bzw. «ethnozentrisch», Vergleiche zwischen Ethnien mit Blick auf ihre Entwicklungshöhe anzustellen.

Trotz dieser Einschränkungen soll im Folgenden der Versuch unternommen werden, die Entwicklung der Patientenrolle resp. des Krankheitsverhaltens im Zuge der abendländischen Kulturevolution nachzuzeichnen.

\section{Ebenen des Altruismus}

Krankenfürsorge wird gemeinhin als kulturelle Errungenschaft betrachtet. Dies darf jedoch nicht darüber hinwegtäuschen, dass das Grundprinzip der Nächstenhilfe bereits im Tierreich vorkommt, von daher tief im Instinktiven verwurzelt und mithin ein Produkt der Evolution des Lebens ist. Nur schon das Koexistieren im Schwarm bietet den beteiligten Lebewesen Flankenschutz vor Fressfeinden [6]. Kooperatives $\mathrm{Zu}$ - 
sammenwirken wie das gemeinsame Jagen von Pelikanen, Wölfen oder Hyänen gereicht den Beteiligten zu unmittelbarem Nutzen, kann dementsprechend nicht als altruistisch bezeichnet werden. Altruistisch können demnach nur Aktionen sein, bei denen ein Individuum einem anderen einen Vorteil verschafft, ohne gleichzeitig ebenfalls $\mathrm{zu}$ profitieren. Dieser Sachverhalt dürfte in ursprünglichster Form in der Mutter-Kind-Beziehung verwirklicht sein. Von dieser elementaren Art der Fürsorge leiten sich laut Eibl-
Nun werden vereinzelt Verhaltensweisen bei Tieren beobachtet, die völlig anders gelagert sind: So kann eine Vogelmutter einen gebrochenen Flügel vortäuschen, um so einen Fressfeind von ihrem Nest abzulenken [12]. Ein solch simulatorisches Verhalten ist nun ganz klar final angelegt, dient nicht mehr nur der eigenen physischen Selbsterhaltung. Damit erinnert es an das weiter unten zu besprechende «abnormal illness behavior».

\section{Von nun an stand im wesentlichen das Kollektiv der Versicherten für das erkrankte Individuum ein, dessen Los dadurch zumindest wirtschaftlich stark gemildert wurde}

Eibesfeldt [7] die weiteren Bindungsarten wie Liebe, Freundschaft und die verschiedenen Altruismusformen ab. Die phylogenetisch älteste Form ist dabei der «nepotistische Altruismus» (Verwandtenunterstützung). Wirklich selbstlos ist dieser nicht, da der Ausübende auf diesem Weg einem Teil seiner eigenen Gene bessere Überlebenschancen für die nächste Generation verschafft, ohne sich selbst fortzupflanzen («inclusive fitness» statt «reproductive fitness»).

Dieser prospektive Belohnungseffekt auf genetischer Ebene findet bei der jüngeren Form des «reziproken Altruismus» nicht statt. Dieser bezeichnet das Prinzip «tit for tat» (kooperiere mit jedem, der erfahrungsgemäss - auch mit dir kooperiert) und bedeutet Kooperation auf Vorschuss über den Kreis der Blutsverwandten hinaus [8]. Dabei ist allerdings noch eine Zwischenstufe auszumachen: Der parochiale Altruismus [9]. Hier wird vorzugsweise nur innerhalb einer kleineren Subpopulation kooperiert, die rassisch, ethnisch, religiös oder weltanschaulich definiert sein kann.

Wenn es nun konkret um krankenfürsorgliches, d. h. altruistisches Verhalten gegenüber geschwächten Artgenossen geht, so finden wir dafür relativ wenige Beispiele bei Tieren (Wundenlecken bei Caniden, Bergung verletzter Delphine, Spontanhilfe unter Ratten). Bei anderen Tieren (Hunde, Elefanten) ist Hilfsbereitschaft offenbar davon abhängig, ob ein Tier selbst schon die Erfahrung des Geholfenwerdens gemacht hat [10].

Für den Verhaltensstil eines erkrankten Tieres ist das sogenannte «sickness behavior» absolut prägend [11]: Durch die Entzündung freigesetzte Interleukine sorgen nicht nur für die reparativen Prozesse im Organismus; vielmehr stellen sie über spezifische Rezeptoren im Zentralen Nervensystem das Tier ruhig. Diese kausal herbeigeführte Schonhaltung ermöglicht die bestmögliche Entfaltung der Selbstheilungskräfte, ist also ein biologisches Programm von hohem Anpassungswert.

\section{Die Verhältnisse beim Menschen}

Über die Krankenpflege beim Menschen der Frühzeit ist recht wenig bekannt. Stellt man auf den Kulturanthropologen Lévy-Bruhl [13] ab, so herrscht bei Naturvölkern diesbezüglich ein starker Fatalismus: Hatte der Schamane einmal den Tod vorausgesagt, so wollte man den Schicksalsmächten nicht in die Quere kommen. Pflege und Begleitung des Sterbenden oblagen den Angehörigen (Verwandtenunterstützung).

In der Antike traten dann gelehrte Ärzte auf, die den Gutsbesitzer-Familien bei der Betreuung ihrer erkrankten Sippenmitglieder, aber auch Bediensteten zur Seite standen [14]. Man könnte darin allenfalls eine parochialistische Entwicklungsstufe sehen.

Im Mittelalter lag die Krankenfürsorge dann vor allem in den Händen der Klöster, wo insbesondere Nonnen für «Gotteslohn» auch Fremde pflegten. Diese Form der Wohltätigkeit kann jedoch noch nicht als «reziproker Altruismus» (Leistung gegen angemessene Gegenleistung) bezeichnet werden.

Reziprok wurde der Altruismus erst dort, wo freiberufliche Ärzte ihre Patienten gegen ein vereinbartes Honorar behandelten. Dabei blieb es allerdings nicht: Zur Finanzierung der Armen-Krankenpflege mussten Krankenkassen geschaffen werden (in Deutschland erstmals nach einer kaiserlichen Botschaft von 1881).

Mit dem Aufkommen dieser Art von Sozialversicherung wurde der reziproke Altruismus zwischen Arzt und Patient durch ein Prinzip abgelöst, welches man als «Solidarismus» bezeichnen könnte. Von nun an stand im wesentlichen das Kollektiv der Versicherten für das erkrankte Individuum ein, dessen Los dadurch zumindest wirtschaftlich stark gemildert wurde: Eine komplette Behandlung war nun für bescheidene Prämienzahlungen erhältlich. Schon sehr früh wurde indessen die Gefahr erkannt, dass diese Leistungen auch missbräuchlich beansprucht werden könnten. So musste man sich beim Bezug des Krankenscheins persönlich den Kassenvertretern vorstel- 
len. Ebenso musste man sich das ärztliche Zeugnis dadurch «verdienen», dass man sich gegenüber dem betreffenden Mediziner verpflichtete, eine entsprechende Behandlung über sich ergehen zu lassen. Mit dieser Disziplinierungslogik versuchte das System, das Verhalten des Patienten dahingehend zu steuern, dass er durch pflichtgemässes Mitarbeiten - durchaus im Sinne der Reziprozität - den Schaden klein zu halten half [15].

Die Entlastung des Einzelnen ging allerdings noch weiter. 1960 wurde in der Schweiz die Invalidenversicherung gegründet, welche die Aufgabe hat, die Existenzgrundlage bei Versicherten mit dauernden Gesundheitsschäden durch Eingliederungsmassnahmen oder Rentenleistungen zu sichern. Dabei behielten zumindest auf dem Papier versicherungsrechtliche Prinzipien wie die Pflichten zur Schadensminderung, Mitwirkung und Selbsteingliederung durchaus ihre Gültigkeit. Angesichts der Überschuldung dieses Sozialwerkes mit weit über einem Dutzend Milliarden Franken, darf allerdings die kritische Frage gestellt werden, ob diesen Grundsätzen auch gebührend nachgelebt wird.

\section{Krankenrolle und Krankheitsverhalten}

Seit längerem ist es den Psychiatern bekannt, dass es Störungsbilder gibt, die auf Täuschungsmanövern beruhen: Man denke an das Ganser-Syndrom, die Hysterie, die Pseudologia phantastica, das MünchhausenSyndrom u.a.m. Solche Verhaltensweisen machen nur Sinn, wenn man sie als zweckgerichtete Botschaften an die soziale Umgebung auffasst. Vom Kranken wird indessen Redlichkeit und Kooperation erwartet. Parsons [16] beschrieb 1951 die «Krankenrolle», wie sie dem Patienten von der Gemeinschaft zugedacht wird: Schadensvermeidung, Aufsuchen von Hilfe, Mitwirkung bei Diagnostik und Behandlung. Im Sinne einer Reziprozität zwischen Individuum und

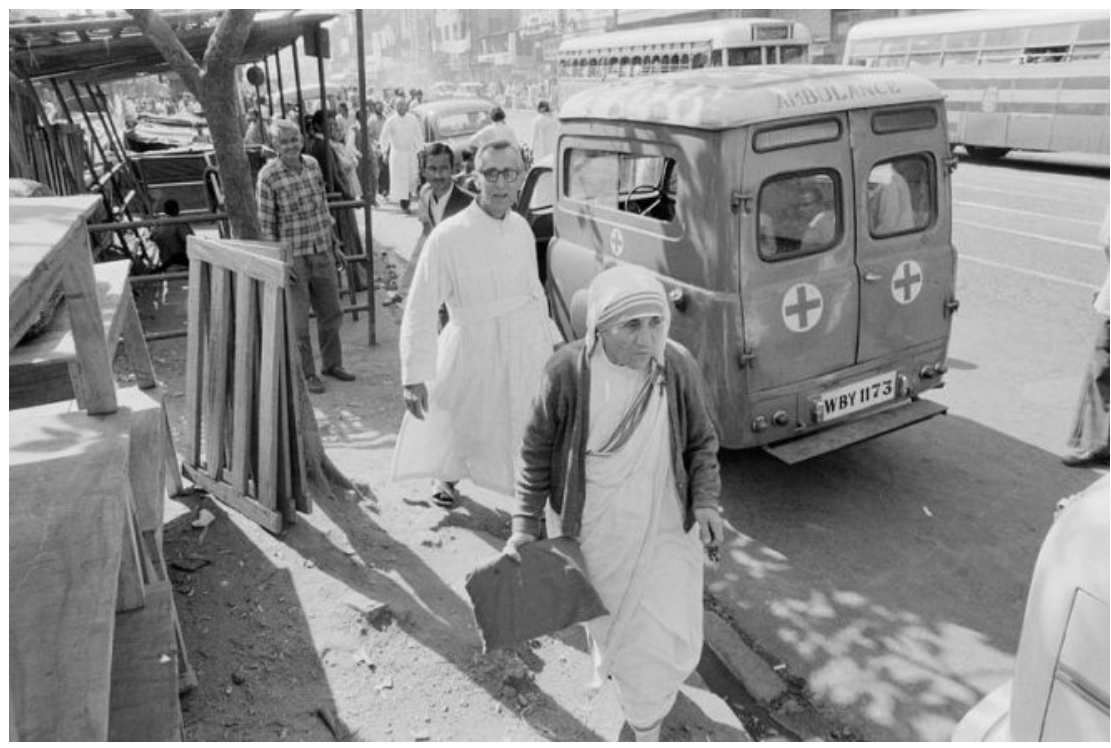

Krankenpflege für «Gotteslohn», bei Klöstern im Mittelalter nicht selten, ist heutzutage die Ausnahme. (Hier Mutter Teresa bei ihrer Arbeit in einem Armenviertel Kalkuttas.)
Kollektiv wird auf grösstmögliche Schadensminderung abgezielt. Es wurde aber bald klar, dass diese Rolle auch auf suboptimale Art gelebt werden kann. Mechanic [17] hielt 1961 fest, dass dieselbe Symptomatik von verschiedenen Menschen sehr unterschiedlich wahrgenommen, interpretiert und ausgelebt werden kann, was wiederum stark von Persönlichkeitsmerkmalen wie Alter, Geschlecht, Sozialstatus und soziokultureller Prägung abhänge. Mit der Frage des «abnormen Krankheitsverhaltens» hat sich dann vor allem Pilowsky [18] auseinandergesetzt. Für ihn war ein solches gegeben, wenn ein Patient trotz objektiv leichter Symptomatik ein schwer betroffenes

\section{Schwierig zu definieren ist die Grenze zwischen systemgewolltem Solidarismus und dem untragbaren} «Hypersolidarismus»

und anhaltendes Leidensbild zeigt, das auch dann nicht verschwindet, wenn alle sachdienlichen Untersuchungen durchgeführt sind und der Patient über die Gutartigkeit seiner Störung aufgeklärt worden ist. Er sah indessen keinen Anlass dazu, wie viele seiner Zeitgenossen von «Hysterie» oder «Hypochondrie» zu sprechen, sondern sah darin vielmehr ein unangemessenes Beharren auf der Krankenrolle und den damit verbundenen Privilegien. Die Psychodynamik, die dann hin zur völligen Chronifizierung führt, beschrieb Weinstein [19]: Für diesen besteht im Vorfeld oftmals eine psychosoziale Problematik, die sich zunehmend zermürbend auf Selbstwertgefühl und Befindlichkeit des Patienten auswirkt. Wird dann ein Abfall der Leistung am Arbeitsplatz festgestellt, so kann diese Selbstwertkrise akzentuiert werden. Eine geringfügige Gesundheitsstörung (z.B. Bagatellunfall, «Verhebetrauma», Befindlichkeitsstörungen) liefert dann die Legitimation für den Rückzug aus dem Leistungsbereich, ohne dass man das Stigma des psychisch Gestörten auf sich lädt. Familiäre und soziale Unterstützungsmechanismen werden mobilisiert (sekundärer Krankheitsgewinn) und tragen das ihrige zur Fixierung dieser Fehlhaltung bei.

Welche spezifischen Verarbeitungspsychismen dabei die dysfunktionale Störungsbewältigung begünstigen, zeigte Knecht [20] in seiner Arbeit von 2008 auf.

\section{Schlussbetrachtungen}

Es wird ohne weiteres deutlich, dass bestimmte Formen des Krankheitsverhaltens nur in einem besonders ausgestalteten Kontext Sinn machen. Von daher hat das Vorhandensein von Sozialversicherungen und weiteren Hilfsangeboten klarerweise einen pa- 
thoplastischen Einfluss auf das individuelle Krankheitsverhalten. So kann ein abnormes Krankheitsverhalten, etwa bei chronischen Schmerzen, durchaus Anpassungswert im Sinne einer Überlebensstrategie erhalten, wie Knecht [21] 2009 dargelegt hat. Der Schmerz transzendiert dann seine Aufgabe der körperlichen Integritätssicherung und wird zum Instrument der sozioökonomischen Selbsterhaltung. Somit hat sich das Krankheitsverhalten, resp. die Beziehung zwischen Individuum und Kollektiv, im Zuge der soziokulturellen Evolution zum Teil deutlich gewandelt. Das Solidaritätsprinzip wurde laufend ausgebaut, was natürlich die Gefahr einer Beanspruchung über Gebühr mit sich brachte. Mithin droht die Gefahr eines «Hypersolidarismus», der auf Dauer das Ende der Sozialwerke bedeuten könnte. Schwierig zu definieren ist die Grenze zwischen systemgewolltem Solidarismus und dem untragbaren «Hypersolidarismus», zumal die finanziellen Aufwendungen noch eine Weile aus anderen Mitteln verstärkt werden können. Juristisch-theoretisch kann sie dort gezogen werden, wo beim Versicherten das sog. «Moral-HazardVerhalten» auftritt. Dieses versicherungswirtschaftliche Konzept erfasst die Fälle, bei denen Versicherungsnehmer nach Vertragsabschluss höhere Risiken in Kauf nehmen, als sie dies ohne Vertrag täten. Dieser Begriff passt demnach auch, wenn ein Individuum seinen Wiedereinstieg am Arbeitsplatz ohne zwingenden medizinischen Grund hinauszögert.

Solidarität als Ausdruck einer gesunden Sozialmoral folgt demnach dem Optimalitäts- und nicht dem Maximalitätsprinzip (was in der Evolutionslehre eine vertraute Erscheinung ist). In der politischen Diskussion wird diesem Umstand nicht immer Rechnung getragen.

So opponierte schon in den 60er Jahren der Anthropologe und Moralphilosoph Arnold Gehlen [21] gegen eine «Hypertrophie der Moral», wobei er geltend machte, dass sich dadurch das Sozialethos immer mehr den Privatinteressen Einzelner unterordne. Mit aussergewöhnlicher Schärfe kommentierte er die extrem hoch angesetzten gesinnungsethischen Positionen von damaligen Intellektuellen, die er unfreundlich als «Mundwerksburschen» bezeichnete. Nach seinem Dafürhalten fehlte diesen hochfliegenden Prinzipien der Realitätsbezug; vielmehr sah er diese als Ausdruck gruppenspezifischer Interessen (namentlich der Publizisten). Man mag ihm recht geben oder auch nicht. Jedenfalls wird die Gemeinschaft im Interesse ihrer Sozialwerke nicht darum herumkommen, ihre Erwartungen hinsichtlich eines zumutbaren Krankheitsverhaltens immer wieder zu reflektieren, $\mathrm{zu}$ formulieren und grobe Widerhandlungen zu sanktionieren.

\section{Literatur}

1 Breuer H. Darwins Theorie erklärt alles. Tagesanzeiger, 24.11.2009, S.48.

2 Zurek WH. Quantum Darwinism and envariance. In: Barrow JD, Davies PCW, Harper CH (Hrsg.). Science and ultimative reality: From quantum to cosmos. Cambrige: Cambridge University Press; 2004. S. 1-17.

3 Smolin L. The life of the cosmos. London: Weidenfeld and Nicolson; 1997.

4 Dawkins R. Das egoistische Gen. Heidelberg: Spektrum Akademischer Verlag; 1994.

5 Blackmore S.: Die Macht der Meme oder die Evolution von Kultur und Geist. Darmstadt: Wissenschaftliche Buchgesellschaft; 2000.

6 Lorenz K. Das sogenannte Böse - Zur Naturgeschichte der Aggression.Wien: Borotha-Schoeler. S. 199-212.

7 Eibl-Eibesfeldt I. Die Biologie menschlichen Verhaltens - Grundriß der Humanethologie. München: Piper; 1984.

8 Trivers RL. The evolution of reciprocal altruism. Q Rev Biol. 1971;46:35-57.

9 Bernhard H, Fischbacher U, Fehr E. Parochial altruism in humans. Natures. 2006;44:912-5.

10 Rankin D. Assortment and the evolution of generalized reciprocity. Evolution. 2009;63(7):1913-22.

11 Hart BL. Biological bases of the behavior of sick animals. Neuroscience \& Biobehavioral Reviews. 1988;12:123-137.

12 Anonymus (online). Überstauwiesen - Der flüssige Wald. http: //de.mongabay.com/rainforest/0605.htm

13 Lévy-Bruhl L. Die geistige Welt der Primitiven. München: Bruckmann; 1927.

14 Hähner-Rombach S. Quellen zur Geschichte der Krankenpflege. Frankfurt a.M.: Mabuse; 2008. S. 19-42.

15 Göckenjan G. Kurieren und Staat machen - Gesundheit und Medizin in der bürgerlichen Welt. Frankfurt a.M.: Suhrkamp; 1985.

16 Parsons T. The social system. New York: Free Press of Glencoe; 1951.

17 Mechanic D. The concept of illness behaviour. J Chron Dis. 1961;8:16-30.

18 Pilowsky I. Abnormal illness behaviour. Br J Med Psychol. 1969;42:347-351.

19 Weinstein MR. The concept of the disability process. Psychosomatics. 1978;19:94-97.

20 Knecht T. Wenn der Schmerzpatient nicht arbeitet Phänomene der dysfunktionalen Beschwerdenbewältigung als Rehabilitationshindernisse. Schweiz Med Forum. 2008;8:797-802.

21 Knecht T. Somatoforme Schmerzstörung - Kann abnormales Krankheitsverhalten adaptiv sein? Schweiz Zschr Psychiatr Neurol. 2009;9:31-34.

22 Gehlen A. Moral und Hypermoral - Eine pluralistische Ethik. Frankfurt a.M.: Athenäum; 1969. 